The AstrophysicAl Journal, 540:588-591, 2000 September 1

(C) 2000. The American Astronomical Society. All rights reserved. Printed in U.S.A.

\title{
AROMATIC MOIETIES IN METEORITES: RELICS OF INTERSTELLAR GRAIN PROCESSES?
}

\author{
M. A. SEPhTON AND I. GILMOUR \\ Planetary Sciences Research Institute, Open University, Milton Keynes, Buckinghamshire, MK7 6AA, England; \\ m.a.sephton@open.ac.uk \\ Received 1999 August 25; accepted 2000 April 3
}

\begin{abstract}
The carbonaceous chondrite meteorites contain a record of the formation of the solar system, part of which is present within organic matter. This organic matter is predominantly aromatic, and its sources remain controversial. The $\delta^{13} \mathrm{C}$ values for individual free and macromolecular aromatic moieties from Cold Bokkeveld and Murchison suggest that these units originate from radiation-induced "circle" reactions involving simultaneous bond synthesis and cracking. Large carbon isotope fractionations and a deuterium enrichment for these entities suggest that these reactions occurred in a dense interstellar cloud. The juxtaposition of the synthesis and cracking products implies that the reactions occurred in a restricting medium, the most likely candidate for which is the icy organic mantles of interstellar grains. In contrast, the $\delta^{13} \mathrm{C}$ record in aromatic moieties from Orgueil is mostly obscured, possibly due to the increased levels of parent body aqueous alteration experienced by this meteorite. These novel observations are consistent with the interstellar-parent body hypothesis, where the final form of meteoritic organic matter results from the transfiguration of interstellar organic precursors by aqueous reactions on the meteorite parent body.

Subject headings: ISM: molecules - meteors, meteoroids - methods: laboratory
\end{abstract}

\section{INTRODUCTION}

The carbonaceous chondrites are a unique class of meteorite that may originate from the C-type asteroids in the outer asteroid belt. These asteroids are ancient and, together with the meteorites derived from them, constitute a record of the birth of the solar system following the collapse of its dense interstellar cloud precursor.

Carbonaceous chondrites can contain up to $5 \%$ of carbon by weight, the majority of which is present as organic matter. Using the Murchison meteorite as an example, over $90 \%$ of the organic matter in carbonaceous chondrites is present as high molecular weight macromolecular material, with the remainder made up of low molecular weight free compounds (Mullie \& Reisse 1987). Aromatic hydrocarbons account for over $5 \%$ of the free organic matter (Mullie \& Reisse 1987) and are genetically related to the dominantly aromatic macromolecular material (Sephton, Pillinger, \& Gilmour 1998). It follows, therefore, that aromatic moieties are quantitatively the most important organic constituents in carbonaceous chondrites.

The dominance of aromatic moieties in carbonaceous chondrites indicates that they are key to understanding the origin of meteoritic organic matter as a whole. The sources of aromatic organic matter in meteorites remain controversial, and circumstellar space (Allamandola, Sandford, \& Wopenka 1987), the solar nebula (Basile, Middleditch, \& Oró 1984; Morgan et al. 1991), and the asteroidal meteorite parent body (Anders 1991) have all been suggested as environments where aromatic organic matter may have been formed.

The most fruitful studies aimed at determining the provenance of extraterrestrial organic molecules have been those that have combined molecular and isotopic information (Yuen et al. 1984; Kerridge, Chang, \& Shipp 1987; Engel, Macko, \& Silfer 1990; Gilmour \& Pillinger 1994; Pizzarello et al. 1991; Cooper et al. 1997; Engel \& Macko 1997; Sephton et al. 1998; Sephton, Pillinger, \& Gilmour 2000a, 2000b; Sephton \& Gilmour 2000). Using this approach, we have been measuring the carbon isotopic composition of individual free and macromolecular aromatic moieties from carbonaceous chondrites (Gilmour \& Pillinger 1994; Sephton et al. 1998, 2000a; Sephton \& Gilmour 2000). Here we have compiled and assessed all of our published and previously unpublished data and may have uncovered evidence for a specific interstellar environment and process for the formation of a significant portion of meteoritic aromatic organic matter.

\section{EXPERIMENTAL}

\subsection{Extraction}

Volatile aromatic hydrocarbons $\left(\mathrm{C}_{7}\right.$ to $\left.\mathrm{C}_{12}\right)$ were extracted using supercritical fluid extraction (SFE) with pure $\mathrm{CO}_{2}$ (90 minute static extraction at 4000 psi, followed by a 45 minute $1 \mathrm{ml}$ minute ${ }^{-1}$ dynamic extraction at 4000 psi) and were collected in diethyl ether cooled to approximately $0^{\circ} \mathrm{C}$ (Sephton et al. 1998, 2000a; Sephton, Pillinger, \& Gilmour 1999).

Less volatile aromatic hydrocarbons $\left(\mathrm{C}_{14}\right.$ to $\left.\mathrm{C}_{22}\right)$ were extracted using solvent extraction ( 2 cycles of ultrasonication with dichloromethane/methanol $85: 15$ for 20 minutes). The extract was concentrated using rotary evaporation and the aromatic compounds isolated by column chromatography $\left(0.5 \times 10 \mathrm{~cm}^{2}, 0.5 \mathrm{~g}\right.$ silica gel column preeluted with $10 \mathrm{ml}$ hexane; the aromatic fraction was eluted with $10 \mathrm{ml}$ toluene).

Aromatic moieties were released from the macromolecular material by thermal dissociation, using both offline hydrous (Sephton et al. 1998, 1999, 2000a) and on-line anhydrous pyrolysis (Sephton \& Gilmour 2000). For offline hydrous pyrolysis, samples were placed in a $1 \mathrm{ml}$ stainless steel insert, and $0.4 \mathrm{ml}$ of high-purity water was added. The insert was then purged with nitrogen gas, sealed, and placed into a $71 \mathrm{ml}$ stainless steel high-pressure reactor (series 4740, Parr Instrument Co.), which was filled with 20 $\mathrm{ml}$ water to minimize pressure differentials. The whole 
arrangement was heated to $320^{\circ} \mathrm{C}$ in a muffle furnace for 72 $\mathrm{hr}$. The released moieties were extracted using SFE as above. For on-line anhydrous pyrolysis, samples were introduced as dry pellets (typically $1 \mathrm{mg}$ ) into an on-line microfurnace (pyrojector, S.G.E. Ltd.) and pyrolyzed at $500^{\circ} \mathrm{C}$ (Sephton \& Gilmour 2000). The pyrojector was directly coupled to the split-splitless injector of the analytical equipment described below.

\subsection{Compound Detection, Identification, and Carbon Isotope Ratio Measurement}

Compound detection and identification was performed by gas chromatography-mass spectrometry (GC-MS) using a Hewlett Packard 5890 gas chromatograph fitted with an HP5 capillary column $(50 \mathrm{~m} \times 0.32 \mathrm{~mm} \times 0.17 \mathrm{~mm})$ and interfaced with a 5971 mass selective detector. Temperature programs were as follows: SFE extracts $-25^{\circ} \mathrm{C}(10$ minutes $), 25^{\circ} \mathrm{C}-220^{\circ} \mathrm{C}\left(5^{\circ} \mathrm{C}\right.$ minute $\left.^{-1}\right), 220^{\circ} \mathrm{C}-300^{\circ} \mathrm{C}$ $\left(10^{\circ} \mathrm{C}\right.$ minute $\left.^{-1}\right)$, and $300^{\circ} \mathrm{C}(12$ minutes $)$; solvent extracts- $50^{\circ} \mathrm{C}(1$ minute $), 50^{\circ} \mathrm{C}-100^{\circ} \mathrm{C}\left(10^{\circ} \mathrm{C}\right.$ minute $\left.^{-1}\right)$, $100^{\circ} \mathrm{C}-300^{\circ} \mathrm{C}\left(5^{\circ} \mathrm{C}\right.$ minute $\left.{ }^{-1}\right)$, and $300^{\circ} \mathrm{C}(14$ minutes); and on-line pyrolysis $-40^{\circ} \mathrm{C}(2$ minutes $), 40^{\circ} \mathrm{C}-300^{\circ} \mathrm{C}\left(5^{\circ} \mathrm{C}\right.$ minute $\left.{ }^{-1}\right)$, and at $300^{\circ} \mathrm{C}$ (6 minutes).

Carbon isotopic measurements for individual compounds in the various extracts were accomplished by gas chromatography-isotope ratio mass spectrometry (GCIRMS). Analyses were performed on a Finnigan MAT Delta S/GC with splitless injection onto an HP5 capillary column $(50 \mathrm{~m} \times 0.2 \mathrm{~mm} \times 0.5 \mathrm{~mm})$. The temperature programs were identical to those used for GC-MS. All obtained carbon isotope ratios are expressed in the $\delta$ notation relative to the international Pee Dee Belemnite (PDB) standard as follows:

$$
\delta^{13} \mathrm{C} \%=\frac{\left({ }^{13} \mathrm{C} /{ }^{12} \mathrm{C}\right)_{\mathrm{sample}}-\left({ }^{13} \mathrm{C} /{ }^{12} \mathrm{C}\right)_{\mathrm{PDB}}}{\left({ }^{13} \mathrm{C} /{ }^{12} \mathrm{C}\right)_{\mathrm{PDB}}} \times 10^{3} .
$$

\section{RESULTS AND DISCUSSION}

Figure 1 displays the $\delta^{13} \mathrm{C}$ values for the free and macromolecular aromatic moieties from Orgueil, Cold Bokkeveld, and Murchison plotted against their carbon number. For Cold Bokkeveld and Murchison, the data points appear to follow a trend, manifested as an arch, where lower molecular weight compounds $\left(C_{7}\right.$ to $C_{8}$ for Cold Bokkeveld; $C_{6}$ to $\mathrm{C}_{11}$ for Murchison) have $\delta^{13} \mathrm{C}$ values that increase with additional carbon atoms, while higher molecular weight compounds $\left(\mathrm{C}_{8}\right.$ to $\mathrm{C}_{14}$ for Cold Bokkeveld; $\mathrm{C}_{11}$ to $\mathrm{C}_{20}$ for Murchison) have $\delta^{13} \mathrm{C}$ values that decrease with increasing carbon number. The higher molecular weight feature is consistent with that seen for $\mathrm{C}_{1}$ to $\mathrm{C}_{5}$ aliphatic hydrocarbons, carboxylic acids, amino acids, and sulphonic acids in Murchison (Yuen et al. 1984; Engel et al. 1990; Pizzarello et al. 1991; Cooper et al. 1997), which has been interpreted as the result of a kinetic isotope effect during the stepwise formation of higher molecular weight compounds from simpler precursors (Yuen et al. 1984). The more reactive ${ }^{12} \mathrm{C}$ is preferentially added during the synthesis of the carbon skeleton of these compounds. If the lower molecular weight trend is also the result of a kinetic isotope effect, then it suggests that bond breaking or "cracking" has produced these compounds. During cracking of the starting material, the more reactive ${ }^{12} \mathrm{C}$ bonds are preferentially broken, leading to lower molecular weight products depleted in ${ }^{13} \mathrm{C}$. Therefore, the aromatic entities seem to have been produced by a
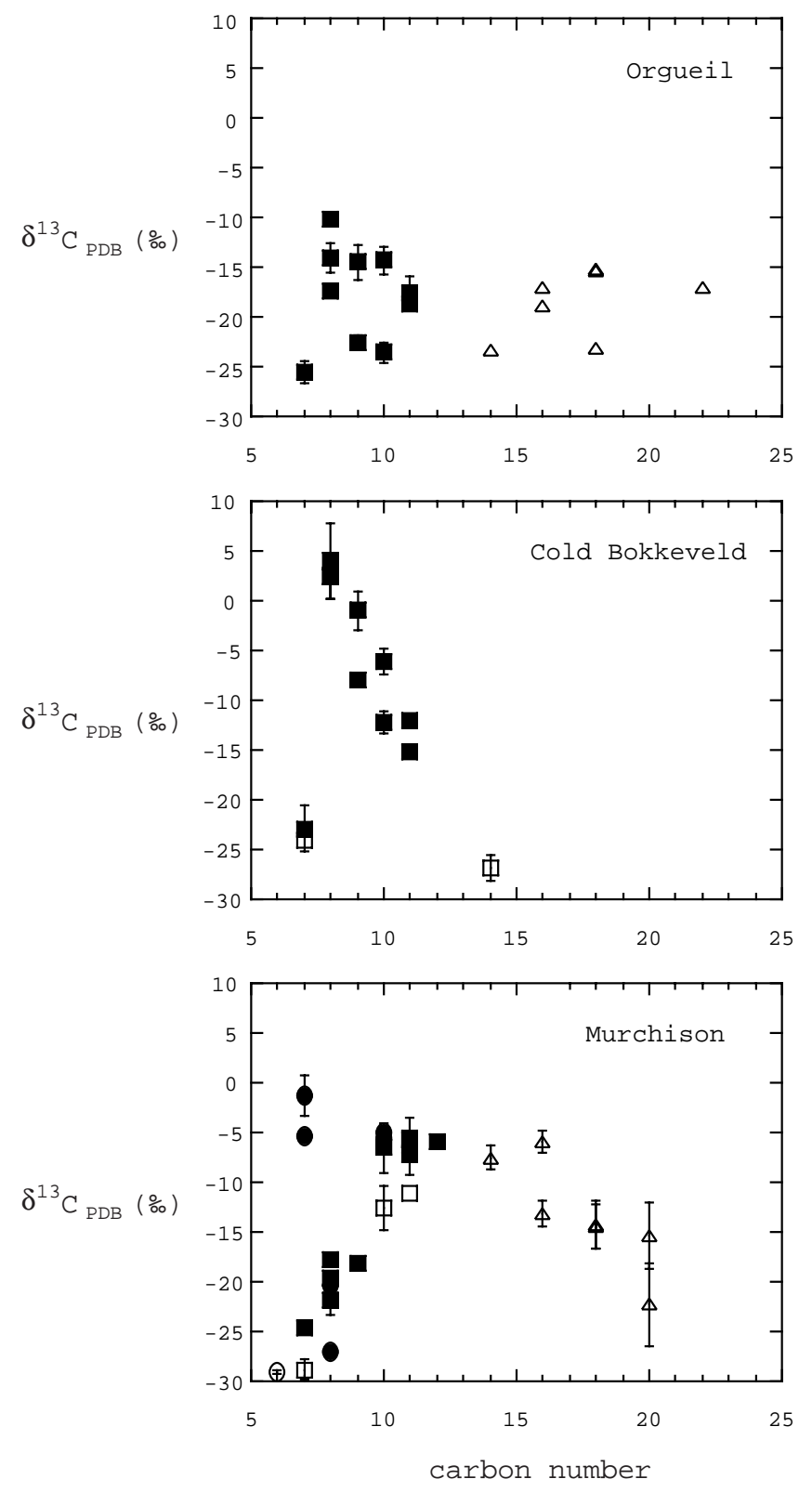

FIG. 1.-Carbon isotopic compositions of individual aromatic hydrocarbon moieties in the Orgueil, Cold Bokkeveld, and Murchison meteorites plotted against their carbon number. Assignments are as follows: benzene released by freeze-thaw disaggregation (open circle), free compounds extracted by supercritical fluid extraction (open square), solventextracted free compounds (open triangle), compounds liberated from the macromolecular materials by off-line hydrous pyrolysis (closed square), and compounds liberated from the macromolecular materials by on-line anhydrous pyrolysis (closed circle). Where available, standard deviations $( \pm 1 \sigma)$ are indicated. For the Murchison free values, $C_{6}$ is from Yuen et al. (1984) and $C_{14}$ to $C_{20}$ are from Gilmour \& Pillinger (1994). The remaining Murchison values are from Sephton et al. (1998) and Sephton \& Gilmour (2000). The Orgueil and Cold Bokkeveld free values were obtained using identical methods to those described in Gilmour \& Pillinger (1994) and Sephton et al. (1998). The macromolecular values for Orgueil and Cold Bokkeveld are from Sephton et al. (2000a).

process in which both bond formation and destruction were significant.

Information about the environment in which this process operated may also be forthcoming from the isotopic data. The $\delta^{13} \mathrm{C}$ values cover a range of more than $20 \%$ for aromatic compounds that differ in size by as few as two carbon 
atoms. During laboratory experiments, where starting materials and products were in the range $\mathrm{C}_{1}$ to $\mathrm{C}_{6}$, synthesis and cracking reactions have produced fractionations of more than 20\% (Chang et al. 1983). However, the isotopic differences between individual products became smaller as their carbon number increased. Therefore, the more than $20 \%$ range in $\delta^{13} \mathrm{C}$ for the relatively large $\mathrm{C}_{6}$ to $\mathrm{C}_{20}$ meteoritic aromatic compounds suggests that during their formation fractionation was at its most extreme.

Kinetic isotope effects are greatest at low temperatures, and of the possible environments for the origin of meteoritic organic matter the lowest temperatures are found in interstellar space. At temperatures as low as $10 \mathrm{~K}$, the molecular products of organic reactions would display large isotopic fractionations, and the fractionation of carbon isotopes does appear to occur in the interstellar medium (Langer et al. 1984; Taylor \& Dickman 1989). Hence, the large range in $\delta^{13} \mathrm{C}$ for the $\mathrm{C}_{6}$ to $\mathrm{C}_{20}$ compounds is consistent with the production of these organic entities in an interstellar environment.

Further evidence for the interstellar provenance of the aromatic moieties in meteorites is provided by their hydrogen isotopic compositions. For Murchison the $\delta \mathrm{D}$ values of bulk free aromatic fractions have been measured at $+244 \%$ to $468 \%$ (Krishnamurthy et al. 1992), while the labile portion of the macromolecular material that releases aromatic compounds upon pyrolysis (Sephton et al. 1998) has $\delta \mathrm{D}$ values as high as $1800 \%$ (Kerridge et al. 1987). These values imply the presence of interstellar molecules in these fractions. Deuterium enrichments have been observed in interstellar clouds (e.g., Jefferts, Penzias, \& Wilson 1973; Hollis et al. 1976) and are generally attributed to large $\mathrm{D} / \mathrm{H}$ fractionations during reactions at the very low temperatures of interstellar space (e.g., Sandford 1996). Although, it should be noted that unimolecular photodissociation reactions may produce Deuterium enrichments in aromatic hydrocarbons without the need for low temperatures (Allamandola et al. 1987).

With an interstellar origin strongly suggested for the aromatic entities, which are found free in the meteorite and can be released from the macromolecular material by pyrolysis, a remaining issue to address is whether the combined molecular and isotopic features for these compounds can constrain the type of reaction that created them. Radiationinduced chemistry plays a major role in creating and modifying organic matter in space. During radiationinduced reactions, organic matter is simultaneously formed and destroyed to achieve an intermediate level of organic complexity (Kissel, Krueger, \& Roessler 1996). The term "circle reaction" has been proposed for this process, and such reactions may have produced and/or modified aromatic hydrocarbons, resulting in the coexisting synthesis and cracking features evident in meteoritic aromatic compounds (Fig. 2). If one portion of organic matter was exposed to more intense or extended periods of radiation than another, then this might produce slight variations in the $\delta^{13} \mathrm{C}$ of their moieties. Different radiation histories may explain why the apexes of the synthesis and cracking features for Cold Bokkeveld and Murchison occur at different carbon numbers in Figure 1. Assessing the scale on which this heterogeneity is significant will require future intra- and intermeteorite studies.

The juxtaposition of aromatic entities created by both synthesis and cracking suggests that, once produced, these

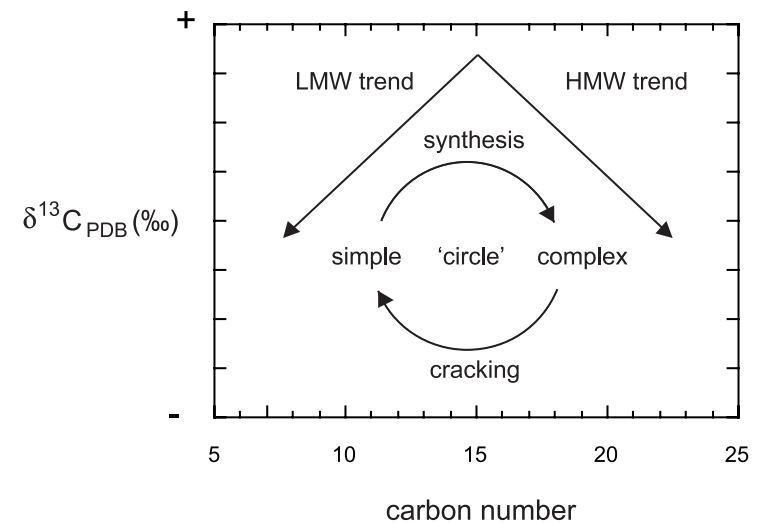

FIG. 2.-Schematic of how radiation-induced circle reactions involve the simultaneous synthesis and cracking of molecules to achieve an intermediate level of organic complexity. This process may be responsible for the $\delta^{13} \mathrm{C}$ vs. carbon number trends in the aromatic hydrocarbons in carbonaceous chondrites. LMW: low molecular weight; HMW: high molecular weight.

compounds were trapped and stored in some way. This observation can be explained if these reactions proceeded in the icy mantles surrounding interstellar grains. At the low temperatures of interstellar space, simple molecules accrete to form icy mantles around interstellar grains, where they are processed by UV and other forms of radiation (Greenberg 1984). The simple molecules are transformed into radical or reactive molecular fragments that can recombine to form larger molecules. Extensive periods of irradiation result in a complex involatile residue comprising aromatic entities and increasing deuterium enrichments (Sandford 1996).

(Poly)aromatic hydrocarbons are the most stable configuration for hydrocarbons because they allow $\pi$ electrons to be delocalized over the entire molecule. This ability to absorb energy would be essential for the survival of the organic matter (Henning \& Salama 1998). Interstellar grains are thought to transition between sheltered dense clouds and the harsh diffuse interstellar medium many times before their ultimate incorporation into a stellar system (Greenberg 1984). It is conceivable that aromatic molecules would become selectively enhanced on grain surfaces as other molecules are destroyed by severe radiation fields present in the diffuse interstellar medium. In this respect, it is interesting to note that the infrared emission features of aromatic hydrocarbons have been detected in the diffuse interstellar medium (Onaka et al. 1996), and aromatic hydrocarbons are considered to be the best candidates for the carriers of the diffuse interstellar bands (e.g., Salama et al. 1996).

In contrast to the better defined trends in Cold Bokkeveld and Murchison, the data points for aromatic entities from Orgueil exhibit more scatter. One possible explanation for this observation is that the structural and isotopic integrity of the aromatic units has been perturbed during secondary processing on the meteorite parent body. Orgueil has experienced more aqueous activity than Cold Bokkeveld and Murchison (Zolensky \& McSween 1988), and the extent of such alteration is believed to have significant effects on the nature of the aromatic organic matter (Wing \& Bada 1991; Sephton et al. 1998, 1999, 2000a).

Therefore, aromatic entities in Cold Bokkeveld and Murchison appear to have isotopic features suggesting an origin 
by radiation-induced circle reactions in the icy mantles of interstellar grains. As the interstellar cloud collapsed to form the solar system, such interstellar grains and their associated aromatic organic matter would have undergone aggregation. These aggregates would have formed chondritic matter in the early solar system, and aliquots of this material would have been preserved in the asteroids. Once on the meteorite parent body, extensive aqueous alteration, such as that experienced by Orgueil, may have partly obscured the isotopic features produced by the circle reactions. These novel interpretations are in harmony with the so-called interstellar-parent body hypothesis (Cronin \& Chang 1993) in which meteoritic organic matter is produced by the aqueous transformation of interstellar precursors initiated by the internal heating of a volatile-rich parent body.

This work was supported by PPARC. The authors thank Scott Sandford for a constructive review.
Allamandola, L. J., Sandford, S. A., \& Wopenka, B. 1987, Science, 237, 56 Anders, E. 1991, Space Sci. Rev., 56, 157

Basile, B. P., Middleditch, B. S., \& Oró, J. 1984, Org. Geochem., 5, 211

Chang, S., Des Marais, D., Mack, R., Miller, S. L., \& Strathearn, G. E. 1983, in Earth's Earliest Biosphere, ed. J. W. Schopf (Princeton: Princeton Univ. Press), 53

Cooper, G. W., Thiemens, M. H., Jackson, T. L., \& Chang S. 1997, Science, 277,1072

Cronin, J. R., \& Chang, S. 1993, in The Chemistry of Life's Origins, ed. J. M. Greenburg, C. X. Mendoza-Gomez, \& V. Pirronello (Dordrecht: Kluwer), 209

Engel, M. H., \& Macko, S. A. 1997, Nature, 389, 265

Engel, M. H., Macko, S. A., \& Silfer, J. A. 1990, Nature, 348, 47

Gilmour, I., \& Pillinger, C. T. 1994, MNRAS, 269, 235

Greenberg, J. M. 1984, Sci. Am., 250, 96

Henning, T., \& Salama, F. 1998, Science, 282, 2204

Hollis, J. M., Snyder, L. E., Lavas, F. J., \& Buhl, D. 1976, ApJ, 209, L83

Jefferts, K. B., Penzias, A. A., \& Wilson, R. W. 1973, ApJ, 179, L57

Kerridge, J. F., Chang, S., \& Shipp, R. 1987, Geochim. Cosmochim. Acta, 51,2527

Kissel, J., Krueger, F. R., \& Roessler, K. 1996, in Comets and the Origin and Evolution of Life, ed. P. J. Thomas, C. P. McKay, \& C. F. Chyba (New York: Springer), 69

Krishnamurthy, R. V., Epstein, S., Cronin, J. R., Pizzarello, S., \& Yuen, G. 1992, Geochim. Cosmochim. Acta, 56, 4045

\section{REFERENCES}

Langer, W. D., Graedel, T. E., Frerking, M. A., \& Armentrout, P. B. 1984, ApJ, 277, 581

Morgan, W. A., Feigelson, E. D., Want, H., \& Frenlach, M. 1991, Science, 252,109

Mullie, F., \& Reisse, J. 1987, Topics Curr. Chem., 139, 85

Onaka, T., Yamamura, I., Tanabe, T., Roellig, T. L., \& Yuen, L. 1996, PASJ, 48, L59

Pizzarello, S., Krishnamurthy, R. V., Epstein, S., \& Cronin, J. R. 1991, Geochim. Cosmochim. Acta, 55, 905

Salama, F., Bakes, E. L. O., Allamandola, L. J., \& Tielens, A. 1996, ApJ, 458,621

Sandford, S. A. 1996, Meteoritics, 31, 449

Sephton, M. A., \& Gilmour, I. 2000, Planet. Space Sci., submitted

Sephton, M. A., Pillinger, C. T., \& Gilmour, I. 1998, Geochim. Cosmochim. Acta, 62, 1821

. 1999, Planet. Space Sci., 47, 181

2000a, Geochim. Cosmochim. Acta, 64, 321

.2000b, Precambrian Res., in press

Taylor, D. K., \& Dickman, R. L. 1989, ApJ, 341, 293

Wing, M. R., \& Bada, J. L. 1991, Geochim. Cosmochim. Acta, 55, 2937

Yuen, G., Blair, N., Des Marais, D. J., \& Chang, S. 1984, Nature, 307, 252

Zolensky, M., \& McSween, H. Y., Jr. 1988, in Meteorites and the Early Solar System, ed. J. F. Kerridge \& M. S. Mathews (Tucson: Univ. Arizona Press), 114 